\title{
Do narcissists benefit from materialistic pursuits? Examining the relation between narcissistic tendencies, extrinsic goals, and well-being
}

\author{
Kaitlyn M. Werner, Aidan Smyth, and Marina Milyavskaya \\ Carleton University
}

\begin{abstract}
Past research consistently finds that the pursuit of extrinsic goals detracts from our happiness and well-being, and instead contributes to ill-being. Despite the robustness of this finding, recent research suggests that people with narcissistic tendencies are more likely to derive meaning from extrinsic goals. We conducted a high-powered $(n=576)$ pre-registered study to examine whether exhibiting higher narcissistic tendencies relates to perceiving materialistic pursuits as being more meaningful and how this in turn influences well-being. Results indicate that having greater narcissistic tendencies was positively associated with perceiving extrinsic goals as being more meaningful, which in turn negatively predicted subjective and eudaimonic well-being, and positively predicted depression. Overall, while it may be the case that people with narcissistic tendencies gain some hedonic benefits from materialistic pursuits, the pursuit of extrinsic goals is negatively related to well-being, as consistent with self-determination theory. All study materials, pre-registration, data, and code can be found at osf.io/8629d.
\end{abstract}

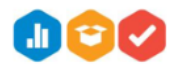

Data, analysis code, supplementary material: osf.io/8629d

Keywords: narcissism, materialism, well-being, extrinsic goals

Modern Capitalist culture tends to emphasize that being rich, famous, and attractive is expected to yield happiness and satisfaction with life. Despite this popular belief, research indicates that the pursuit and attainment of extrinsic life goals for money, fame, and image negatively contributes to well-being, and instead contributes to ill-being (e.g., depression, anxiety; Kasser \& Ryan, 1996; Niemiec, Ryan, \& Deci, 2009).

Correspondence concerning this paper should be addressed to Kaitlyn M. Werner, Department of Psychology, University of Toronto, Toronto, ON, Canada. Email: kaitlyn.werner@utoronto.ca Website: www.kaitlynwerner.wix.com/research

Author note: This work was conducted while KMW was a $\mathrm{PhD}$ student under the supervision of MM in the Department of Psychology at Carleton University. KMW is now a SSHRC Banting Postdoctoral Research Fellow in the Department of Psychology at the University of Toronto.

Author contributions: KMW designed the research question and analyses with feedback from MM. KMW and MM collected the data, which was funded by MM. Data analysis was conducted by KMW. KMW wrote the introduction, methods, and results with feedback from MM. KMW and AS wrote the discussion collaboratively with feedback from MM.

Funding: Preparation of this manuscript was supported by an Insight Grant from the Social Sciences and Humanities Research Council (SSHRC) awarded to MM.
However, recent research suggests that people with narcissistic tendencies are more likely to derive meaning from these more materialistic pursuits (Abeyta, Routledge, \& Sedikides, 2017), therefore suggesting that such goals may be beneficial in certain contexts or for individuals with certain personality traits. This stands in contrast to previous research in self-determination theory, which finds that extrinsic goals are detrimental, even in contexts where such goals are prevalent, and may even be actively promoted and encouraged (e.g., among business students, Vansteenkiste, Duriez, Simons, \& Soenens, 2006; and law students, Sheldon \& Krieger, 2004). In order to reconcile these competing ideas, the purpose of the research is to replicate the finding that extrinsic goals are perceived as meaningful to individuals with narcissistic tendencies, as well as extend this finding by assessing indicators of well-being.

According to self-determination theory (Deci \& Ryan, 2000; Ryan \& Deci, 2017), there are two distinct types of life goals - intrinsic and extrinsic - that people pursue throughout their lives. Intrinsic goals include striving for personal growth, fostering mutual close relationships with others, and being involved with one's community, whereas extrinsic goals focus on the 
excessive accumulation of wealth, maintaining an attractive image, and enhancing one's social status via popularity and/or fame. Individuals who value and pursue more intrinsic (relative to extrinsic) goals are more likely to experience greater well-being (Kasser, 2002; Kasser \& Ryan, 1993; Sheldon \& Kasser, 1998), less distress and higher self-esteem (Kasser \& Ryan, 1996), and engage in less maladaptive behaviours such as drug use and risky sexual behaviour (Kasser \& Ryan, 1993; Williams et al., 2000), whereas the reverse is true for individuals who disproportionately value extrinsic goals (e.g., they experience greater anxiety and depression; Niemiec et al., 2009).

While there are certainly individual differences in the extent to which people generally pursue intrinsic and extrinsic goals (e.g., some people value more extrinsic than intrinsic goals and vice-versa for another person), it should be no real surprise that people with narcissistic tendencies may disproportionally value (Kasser \& Ryan, 1996) or find meaning in the pursuit of extrinsic goals. Narcissism is characterized by grandiosity, self-aggrandizement and status-seeking, and volatile self-esteem (Morf \& Rhodewalt, 2001). Narcissists are also preoccupied with their appearance (Vazire, Naumann, Rentfrow, \& Gosling, 2008) and tend to be more materialistic (Lee \& Seidle, 2012; Rose, 2007). Extending the environmental-match hypothesis regarding values (Sagiv \& Schwartz, 2000) to personality characteristics, one could theoretically reason that people with narcissistic tendencies may benefit from the attainment of extrinsic goals given their congruence with the individual's personality. However, research indicates that this is likely not the case. Vansteenkiste and colleagues (2006) found that, even though business students ascribed greater importance to extrinsic relative to intrinsic goals (compared to education students), the pursuit of such goals was positively related to internal distress and substance abuse, and negatively related to well-being. Similar findings were found in other business student samples (e.g., Kasser \& Ahuvia, 2002), as well as among lawyers (Sheldon \& Krieger, 2004).

In line with these previous findings, Abeyta and colleagues (2017) do indeed address this limitation by saying "...it is possible that narcissists are motivated to pursue extrinsic goals because they view them as meaningful, but these pursuits might ultimately lead them to feel unhappy, be less satisfied with their lives, and place them at risk for psychopathology" (p. 224). While this idea is clearly consistent with past research on extrinsic goals, it is imperative that such ideas be empirically tested. Therefore, in line with previous research examining the outcomes associated with the pursuit and attainment of extrinsic goals (e.g., Niemiec et al., 2009), we chose to focus on measures related to both well-being (subjective and eudaimonic wellbeing) and ill-being (depression). While eudaimonic well-being has yet to be examined with respect to extrinsic goals, it is postulated to be a core aspect of human functioning and wellness, as emphasis is placed on meaning and self-realization rather than hedonic pleasure (e.g., Huta \& Ryan, 2010). More importantly for the current study, meaning in life has been shown to positively relate to eudaimonic functioning (Steger, Kashdan, \& Oishi, 2008); therefore, if people with narcissistic tendencies do indeed perceive extrinsic goals as being meaningful, these goals would theoretically then be positively associated with eudaimonic well-being.

In addition to extending the work of Abeyta and colleagues (2017) by including measures of well-being, we also believe it is important to address a few methodological concerns, some of which were raised as limitations in the original study. First, the traditional Aspirations Index (Kasser \& Ryan, 1996) assesses three types of extrinsic goals - money, fame, image which typically have a moderate to strong correlation with one another (e.g., .51 to .59; Niemiec et al., 2009). However, in the previous study, only two goals were assessed - money and fame. Therefore, our goal was to improve upon the previous method by including a more comprehensive measure that includes all goal types. This is especially important in the context of narcissism, as the goal to maintain an attractive image may be of particular importance to people with high narcissistic tendencies (e.g., Stucke \& Sporer, 2002). Second, the primary analysis in the previous study examined the effect of narcissism and individual goal types (i.e., wealth, image, community, etc.) in predicting perceived meaning. From both a theoretical and empirical perspective, this is problematic because it can be expected that, by and large, all people pursue both intrinsic and extrinsic goals to some degree (Grouzet et al., 2005; Kasser, 2002), and extrinsic goals generally only become problematic when an individual places greater emphasis on such materialistic pursuits at the expense of intrinsic goals. Indeed, a recent metaanalysis examining the association between materialism and well-being found that measures focusing solely on money were weak predictors of well-being (effect sizes ranging from -.07 to -.14), whereas more comprehensive measures of materialism demonstrated a stronger relation (effect sizes ranging from -.16 to -.24; Dittmar, Bond, Hurst, \& Kasser, 
2014). Consistent with this idea, Abeyta and colleagues did indeed find that exhibiting greater narcissistic tendencies was positively associated with perceiving extrinsic (relative to intrinsic) goals as being more meaningful. Therefore, in order to best understand the role of extrinsic goals in the life of a person with narcissistic tendencies (especially as it pertains to wellbeing), it would be pertinent for the current study to replicate and extend this finding instead of focusing on the analyses examining individual goal types.

In sum, in the current pre-registered study we sought to examine the extent to which people with narcissistic tendencies perceive extrinsic (relative to intrinsic) goals as being meaningful (conceptually replicating Abeyta et al., 2017), and how this in turn is associated with various indicators of well-being (extending the previous study by linking it to research conducted within self-determination theory; e.g., Sheldon \& Krieger, 2004; Vansteenkiste et al., 2006).

\section{Pre-Registered Hypotheses}

Consistent with Abeyta and colleagues (2017), we did indeed expect that narcissistic tendencies would be positively associated with perceiving extrinsic (relative to intrinsic) goals as being more meaningful. However, keeping in line with self-determination theory, we expected that perceiving extrinsic (relative to intrinsic) goals would be negatively associated with well-being and positively associated with ill-being. Specifically, we hypothesize that higher narcissistic tendencies will be positively associated with perceiving extrinsic (relative to intrinsic) goals as being more meaningful, which in turn will negatively predict both subjective and eudaimonic well-being, as well as positively predict depression.

\section{Statement of Transparency}

All research questions and hypotheses, analytic strategy, and a draft of the statistical code were preregistered on the Open Science Framework prior to collecting any data. Additionally, all study materials, code, data, and amendments documenting any changes

\footnotetext{
1 We compared the group affected by this glitch to those who did not and there were no significant differences on any of the key study variables (see output on OSF).

2 The details of the power analysis can be found on the project OSF page. The imputed information (i.e., the anticipated means, correlations, etc.) was based on a pilot study.
}

pertaining to this study are also available. Project link: https://osf.io/8629d/

Important changes made to the pre-registered protocol should be mentioned. First, there was a glitch in the Qualtrics system resulting in two duplicate items being presented in the narcissism questionnaire, thus resulting in 14 unique items instead of the expected 16. We did correct this issue, however it affected 93 participants from the final sample, and so we recruited an additional 100 participants to help reduce any bias associated with this error1. Second, we initially registered that we would calculate a depression score by using the sum (as recommended by Eaton, Smith, Ybarra, Muntaner, \& Tien, 2004). However, for ease of interpretation in the mediation analysis, we report the mean.

Finally, it should be noted that although we use mediation models to test our hypotheses, we acknowledge that we cannot infer any causal conclusions. However, because these models stem directly from previous research (e.g., Abeyta et al., 2017), we believe there is at least some theoretical merit to support the proposed directionality of the different variables. Nonetheless, no causal inference should be drawn from these models.

\section{Methods}

\section{Participants and Procedure}

Based on a power analysis using Shoemann, Boulton, and Short's (2017) ShinyApp for running Monte Carlo simulations for mediation models, it was determined that we needed to recruit a minimum of 430 participants to detect the hypothesized indirect effects with $95 \%$ power2. As per our pre-registration, we initially rounded up to 500 , but due to the aforementioned technical glitch in the survey, we collected data from 6423 conveniently sampled adults recruited online using Turk Prime (Litman, Robinson, \& Abberbock, 2016). As part of a larger survey, participants completed measures of narcissistic tendencies, intrinsic and extrinsic goals, and wellbeing. Based on our pre-registered exclusion criteria, 51 participants were removed for completing the

\footnotetext{
3 We initially aimed to collect data from an additional 100 participants; however, due to the MTurk recruitment method (i.e., they keep collecting data until you receive the specified number of complete hits), there were an additional 42 participants who did not fully complete the survey and were thus not counted by MTurk. Most of these responses were filtered out using the preregistered data-screening methods.
} 
survey in less than five minutes and eight participants were removed for failing to pass at least two of three attention checks. Additionally, we removed three duplicate responses and four participants for having no responses for any of the attention checks (indicating that they did not complete a significant portion of the questionnaire). Thus, our final sample consists of 576 participants4. This study was approved by the Carleton University Research Ethics Board - B (for science, health, engineering, design, psychology, and cognitive science). Details about the ethics protocol (including the specific protocol number, date of approval, etc.) can be found on the participant consent form provided in the project link above.

\section{Measures}

Narcissistic Tendencies. Consistent with the original study (e.g., Abeyta et al., 2017), we used the Narcissistic Personality Inventory (NPI-16; Ames, Rose, \& Anderson, 2006) to assess the extent to which participants exhibit narcissistic tendencies. The NPI-16 is a forced-choice measure where participants are presented with two different items and are asked to select which of the pair "comes closest to describing your feelings and beliefs about yourself." In each pair, one item is narcissistic (e.g., "I am more capable than other people") and the other is more general (e.g., "There is a lot that I can learn from other people"). Narcissistic responses were coded as 1 and the other response as 0 . A total score was calculated by summing the response to all 16 items, with higher scores indicating the exhibition of greater narcissistic tendencies. Cronbach's alpha was 0.80 .

Relative Extrinsic Value Orientation (REVO) Meaning and Importance. We adapted the Aspirations Index (35-items; Kasser \& Ryan, 1996) to assess the extent to which intrinsic and extrinsic life goals are perceived as both meaningful and important. Intrinsic goals consist of self-acceptance, having meaningful relationships, and community contributions, whereas extrinsic goals include wealth, image, and popularity. Each of the six life goals has five corresponding items, and responses were made on a 1 (not at all) to 5 (extremely) Likert scale. We calculated a relative extrinsic value orientation (REVO) index for

\footnotetext{
4 We would like to acknowledge that we did not collect any demographic data for this study (it was an oversight, but also not necessarily needed for the purposes of the present research) and so not including this information in the manuscript is not an error of omission. Participants are comprised of MTurk recruits from the United States and Canada, and so general information about the
}

both meaning and importance by subtracting the average of all intrinsic items from the average of all extrinsic items, such that higher scores indicate that extrinsic goals are considered as being more meaningful or more important than intrinsic goals. Cronbach's alpha for both the meaning and importance subscales range from 0.91 to 0.94 .

Eudaimonic Well-Being. The Questionnaire for Eudaimonic Well-Being (21-items; Waterman et al., 2010) assesses the extent to which an individual exhibits eudaimonic functioning, such as selfrealization, developing one's potentials, and living a purposeful life. Responses were made on a 5-point Likert scale, ranging from 0 (strongly disagree) to 4 (strongly agree). A mean score was calculated by averaging all items, such that higher scores indicate greater experiences of eudaimonic well-being. Cronbach's alpha for this scale was 0.87 .

Depression. The Centre for Epidemiologic Studies Depression Scale - Revised (CESD-R; Eaton et al., 2004) was used as an indicator of ill-being (e.g., Niemiec et al., 2009). Participants were asked to rate the extent to which they experienced a list of 20 feelings and behaviours over the past two weeks. Responses were made on a 5-point Likert scale, ranging from 1 (not at all or less than 1 day) to 5 (nearly every day for 2 weeks). A mean score was calculated by averaging all items, with higher scores indicating greater levels of depression. Cronbach's alpha was 0.96 .

\section{Results}

\section{Pre-Registered Analyses}

Descriptive statistics and correlations among all study variables are presented in Table 1 . To test our pre-registered hypotheses, we conducted simple mediation analyses using ordinary least squares path analysis (Hayes, 2013). Specifically, we independently examined the relation between narcissistic tendencies, perceived meaningfulness of extrinsic (relative to intrinsic) goals, and three indicators of well-being subjective well-being, eudaimonic well-being, and depression. Unstandardized coefficients are presented for all analyses, and a detailed summary of all results

demographics of this kind of sample can be found in Difalla, Filatova, and Ipeirotis (2018) who conducted a study assessing the demographics of nearly 40,000 MTurk participants. Specifically, within the US, participants tend to be evenly split in terms of gender (females $=55 \%$ ) with ages ranging between 18-67 years old. 
Table 1.

Descriptive statistics and correlations between narcissistic tendencies and the different compositions of intrinsic and extrinsic goals $(n=576)$.

\begin{tabular}{|c|c|c|c|c|c|c|c|c|c|c|c|c|c|c|c|c|c|c|c|}
\hline & & $M$ & $S D$ & 1 & 2 & 3 & 4 & 5 & 6 & 7 & 8 & 9 & 10 & 11 & 12 & 13 & 14 & 15 & 16 \\
\hline 1 & Narcissism & 3.75 & 3.37 & -- & & & & & & & & & & & & & & & \\
\hline 2 & $\mathrm{REVO}^{\mathrm{a}}$ & -2.30 & 1.58 & .47 & -- & & & & & & & & & & & & & & \\
\hline 3 & Intrinsic ${ }^{b}$ & 5.72 & 0.98 & -.17 & -.57 & - & & & & & & & & & & & & & \\
\hline 4 & Personal growth & 5.90 & 0.98 & -.10 & -.44 & .84 & -- & & & & & & & & & & & & \\
\hline 5 & Community & 5.32 & 1.35 & -.13 & -.46 & .85 & 0.57 & -- & & & & & & & & & & & \\
\hline 6 & Relationships & 5.95 & 1.16 & -.19 & -.53 & .83 & 0.60 & 0.50 & -- & & & & & & & & & & \\
\hline 7 & Extrinsic $^{c}$ & 4.23 & 1.30 & .44 & .79 & .06 & 0.10 & 0.08 & -0.03 & -- & & & & & & & & & \\
\hline 8 & Money & 4.06 & 1.53 & .36 & .66 & .05 & 0.16 & -0.02 & 0.00 & 0.84 & -- & & & & & & & & \\
\hline 9 & Fame & 2.93 & 1.50 & .43 & .68 & .05 & 0.06 & 0.12 & -0.06 & 0.87 & 0.56 & - & & & & & & & \\
\hline 10 & Image & 3.28 & 1.51 & .35 & .69 & .06 & 0.03 & 0.10 & -0.01 & 0.88 & 0.60 & 0.68 & -- & & & & & & \\
\hline 11 & Subjective well-being & 4.48 & 2.13 & .10 & -.15 & .22 & 0.13 & 0.19 & 0.24 & -0.01 & -0.06 & 0.02 & 0.01 & - & & & & & \\
\hline 12 & Positive affect & 3.04 & 0.88 & .18 & -.06 & .28 & 0.22 & 0.29 & 0.20 & 0.14 & 0.10 & 0.16 & 0.12 & 0.73 & -- & & & & \\
\hline 13 & Negative affect & 1.74 & 0.82 & .03 & .13 & -.06 & -0.05 & -0.01 & -0.10 & 0.11 & 0.10 & 0.09 & 0.09 & -0.69 & -0.24 & -- & & & \\
\hline 14 & Life satisfaction & 3.19 & 1.09 & .08 & -.15 & .16 & 0.03 & 0.13 & 0.23 & -0.06 & -0.11 & -0.03 & -0.02 & 0.85 & 0.44 & -0.40 & -- & & \\
\hline 15 & Depression & 1.75 & 0.83 & -.02 & .11 & -.08 & -0.07 & -0.03 & -0.10 & 0.07 & 0.09 & 0.04 & 0.05 & -0.68 & -0.36 & 0.79 & -0.46 & -- & \\
\hline 16 & Eudaimonic well-being & 2.55 & 0.57 & -.04 & -.45 & .48 & 0.45 & 0.37 & 0.38 & -0.18 & -0.19 & -0.12 & -0.16 & 0.59 & 0.50 & -0.38 & 0.45 & -0.42 & - \\
\hline
\end{tabular}

Note aREVO $=$ Extrinsic - Intrinsic. bntrinsic $=$ Composite of all three intrinsic goals. cExtrinsic $=$ Composite of all three extrinsic goals. Correlations greater than .14 are $p<.001$, greater than .10 are $p<.05$, and greater than .10 are $p<.10$.

can be found in Table 2. Consistent with our hypotheses, results indicate that people who exhibited greater narcissistic tendencies perceived extrinsic (relative to intrinsic) goals as being more meaningful $(a=.22, p<.001,95 \%$ CI $[.19, .25])$, which in turn was negatively associated with both subjective well-being $(b=-.34, p<.001,95 \%$ CI [-.47, -.22]) and eudaimonic well-being $(b=-.20, p<.001,95 \%$ CI [-.23, -.17]), and was positively associated with depression $(b=.08, p=$ $.002,95 \%$ CI $[.03, .13])$. Based on 10,000 samples, the biased-corrected bootstrap confidence interval for the indirect effect for each mediation analysis was statistically significant $\left(a b_{s w b}=-.08,95 \%\right.$ CI: [-.11, $.05] ; a b_{e w b}=-.04,95 \%$ CI: [-.05, -.04]; $a b_{\text {dep }}=.35$, $95 \%$ CI: $[.12, .58])$. Importantly, there was a significant total effect of narcissistic tendencies on subjective well-being ( $c=.07, p=.013,95 \%$ CI $[.01, .12])$, which was further magnified when accounting for perceptions of extrinsic (relative to intrinsic) goals $\left(c^{\prime}=.14, p<\right.$ $.001,95 \%$ CI $[.08, .20])$. This provides evidence of a suppression effect, as the total effect is closer to zero than the direct effect, and the direct and indirect effects are of the opposite sign (MacKinnon, Krull, \& Lockwood, 2000). As per a reviewer suggestion, we also included a set of analyses assessing perceived meaning of intrinsic and extrinsic goals as separate

5 With respect to the valuing of intrinsic and extrinsic life goals, the central premise within self-determination theory is that the pursuit of more extrinsic goals only becomes problematic when they start to outweigh or impede a person's intrinsic life goals. This is why we initially pre-registered a composite score rather than looking at the different goals as separate mediators This use of relative scores is also consistent with previous research within the values literature, which proposes that the relative importance mediators (see post hoc analyses in Table 2). Although the results are fairly consistent (with the exception of depression), it is important to note that the use of the composite score is more relevant in the context of the present research (as we pre-registered) 5 .

\section{Exploratory Analyses}

In the original study, an adapted version of the Aspirations Index was used, such that only two intrinsic (close relationships, community involvement) and two extrinsic (money, fame) goals were assessed. In the present study, we included the full measure and so we wanted to examine the relation between narcissistic tendencies and the more comprehensive list of goals, especially since the missing extrinsic goal, image, seems especially relevant in the context of narcissism. As indicated in Table 1, correlation analyses indicate that all of the extrinsic goals were positively correlated with another (r's ranging from .56-.68), as did the intrinsic goals (r's ranging from .50-.60). Additionally, narcissism was also moderately and positively associated with all of the extrinsic goals individually, including image ( $r$ 's range from .35 to .42 ).

of a goal is the most informative measure to use (vs. the absolute value of the individual goal of interest) when assessing the extent to which a person cares about a particular value or goal (e.g., Kasser \& Ryan, 1993; Kasser et al., 2014; Rokeach, 1973, Schwartz, 1992). 
Table 2.

Results of pre-registered and post-hoc mediation analyses with intrinsic and extrinsic goals mediating the relation between narcissistic tendencies and indicators of well-being.

\begin{tabular}{|c|c|c|c|c|c|c|c|c|c|c|c|c|c|c|c|}
\hline \multirow[b]{2}{*}{ Path } & \multicolumn{5}{|c|}{$y_{1}:$ Subjective Well-Being } & \multicolumn{5}{|c|}{$y_{2}:$ Eudaimonic Well-Being } & \multicolumn{5}{|c|}{$y_{3}:$ Depression } \\
\hline & b & SE & $\mathrm{t}$ & $p$ & $95 \% \mathrm{CI}$ & b & SE & Est/SE & $p$ & $95 \% \mathrm{CI}$ & $\mathrm{b}$ & SE & Est/SE & $p$ & $95 \% \mathrm{CI}$ \\
\hline \multicolumn{16}{|l|}{ Pre-Registered Analyses } \\
\hline a: Narcissism $\rightarrow$ REVO & .22 & .17 & 12.66 & $<.001$ & {$[0.19,0.25]$} & .22 & .02 & 12.65 & $<.001$ & {$[0.19,0.25]$} & .22 & .02 & 12.71 & $<.001$ & {$[0.19,0.25]$} \\
\hline $\mathrm{b}: \mathrm{REVO} \rightarrow y_{\mathrm{i}}$ & -.34 & .06 & -5.50 & $<.001$ & {$[-0.47,-0.22]$} & -.20 & .02 & -13.06 & $<.001$ & {$[-0.23,-0.17]$} & .08 & .02 & 3.17 & .002 & {$[0.03,0.13]$} \\
\hline $\mathrm{c}^{\prime}:$ Narcissism $\rightarrow y_{\mathrm{i}}$ & .14 & .03 & 4.85 & $<.001$ & {$[0.08,0.20]$} & .04 & .01 & 5.16 & $<.001$ & {$[0.02,0.05]$} & -.02 & .01 & -1.96 & .050 & {$[-0.05,0.00]$} \\
\hline c: Total Effect & .07 & .03 & 2.49 & .013 & {$[0.01,0.12]$} & -.01 & .01 & -0.99 & .319 & {$[-0.02,0.01]$} & -.01 & .01 & -0.53 & .598 & {$[-0.03,0.01]$} \\
\hline ab: Indirect Effect & -.08 & .01 & - & - & {$[-0.11,-0.05]$} & -.04 & .01 & -- & - & {$[-0.05,-0.03]$} & .02 & .01 & -- & - & {$[0.01,0.03]$} \\
\hline \multicolumn{16}{|l|}{ Post-Hoc Analyses } \\
\hline $\mathrm{a}_{1}:$ Narcissism $\rightarrow$ Intrinsic & -.05 & .01 & -4.21 & $<.001$ & {$[-0.07,-0.03]$} & -.05 & .01 & -4.31 & $<.001$ & {$[-0.08,-0.03]$} & -.05 & .01 & -4.23 & $<.001$ & {$[-0.07,-0.02]$} \\
\hline$a_{2}:$ Narcissism $\rightarrow$ Extrinsic & .17 & .01 & 11.59 & $<.001$ & {$[0.14,0.20]$} & .17 & .01 & 11.52 & $<.001$ & {$[0.14,0.20]$} & .17 & .01 & 11.64 & $<.001$ & {$[0.14,0.20]$} \\
\hline $\mathrm{b}_{1}:$ Intrinsic $\rightarrow y_{\mathrm{i}}$ & .58 & .09 & 6.42 & $<.001$ & {$[0.40,0.76]$} & .31 & .02 & 14.44 & $<.001$ & {$[0.26,0.35]$} & -.08 & .04 & -2.34 & .020 & {$[-0.16,-0.01]$} \\
\hline $\mathrm{b}_{2}:$ Extrinsic $\rightarrow y_{\mathrm{i}}$ & -.20 & .07 & -2.62 & .009 & {$[-0.34,-0.05]$} & -.13 & .02 & -7.36 & $<.001$ & {$[-0.16,-0.09]$} & .07 & .03 & 2.49 & .013 & {$[0.02,0.13]$} \\
\hline $\mathrm{c}^{\prime}:$ Narcissism $\rightarrow y_{\mathrm{i}}$ & .13 & .03 & 4.40 & $<.001$ & {$[0.07,0.19]$} & .03 & .01 & 4.47 & $<.001$ & {$[0.02,0.04]$} & -.02 & .01 & -1.92 & .056 & {$[-0.05,0.00]$} \\
\hline c: Total Effect & .07 & .03 & 2.49 & .013 & {$[0.14,0.12]$} & -.01 & .01 & -1.00 & .319 & {$[-0.02,0.01]$} & -.01 & .01 & -0.53 & .598 & {$[-0.03,0.01]$} \\
\hline$a b_{1}:$ Indirect Effect (intrin) & -.03 & .01 & - & - & {$[-0.05,-0.01]$} & -.02 & .00 & -- & - & {$[-0.02,-0.01]$} & .00 & .00 & -- & -- & {$[0.00,0.01]$} \\
\hline $\mathrm{ab}_{2}$ : Indirect Effect (extrin) & -.03 & .01 & - & - & {$[-0.06,-0.01]$} & -.02 & .00 & -- & - & {$[-0.03,-0.02]$} & .01 & .01 & -- & -- & {$[0.00,0.02]$} \\
\hline
\end{tabular}

Note. Unstandardized coefficients are reported.

We also found that narcissistic tendencies were negatively related to all intrinsic goals, albeit to a lesser extent ( $r$ 's range from -.10 to -.19).

Also related to measurement, we were curious about the way perceived meaningfulness of intrinsic and extrinsic goals was assessed in the original study. Traditionally, the Aspirations Index is used to assess the amount of importance people place on a variety of life goals; however, in the original study this scale was adapted to assess whether a person derives any meaning from these goals. To us, how "meaningful" a goal is to a person is likely just a proxy measure of importance (indeed, within self-determination theory, these two constructs are often described together), and may not reflect whether or not a person will derive any sense of real meaning in life from these goals. In other words, just because a person finds something is important and/or meaningful doesn't necessarily mean that it will translate to positive benefits, as people are often misguided in their goal pursuits (Sheldon, Gunz, Nichols, \& Ferguson, 2010; Werner \& Milyavskaya, 2018). Results indicate that the REVO composites for perceived meaningfulness and perceived importance were practically identical, $r(567)=.94, p<.001$. The full correlation matrix between these two measures can be found in the supplementary analyses on OSF.

\section{Discussion}

The purpose of the present study was to examine whether narcissistic tendencies are associated with perceiving extrinsic (relative to intrinsic) goals as being meaningful, and how this predicts various indicators of well-being. Consistent with Abeyta and colleagues (2017), we hypothesized that having higher levels of narcissistic tendencies would be positively

associated with perceiving extrinsic (relative to intrinsic) goals as being more meaningful. However, consistent with the self-determination theory perspective on the pursuit of intrinsic and extrinsic goals, we further hypothesized that perceiving extrinsic (relative to intrinsic) goals as being more meaningful may actually be negatively associated with well-being. Results from a series of mediation analyses provide general support for these hypotheses, specifically finding that exhibiting greater narcissistic tendencies was positively associated with perceiving extrinsic (relative to intrinsic) goals as being more meaningful, which in turn negatively predicted both subjective and eudaimonic well-being, as well as positively predicted depression.

\section{Theoretical Considerations}

From a theoretical perspective, the present findings can be understood in the context of self-determination theory, which posits that extrinsic goals are associated with negative outcomes because they distract from intrinsic pursuits and therefore one's ability to satisfy basic psychological needs (Kasser, Cohn, Kanner, \& Ryan, 2007). Individuals with higher narcissistic tendencies appear to be more invested in extrinsic (relative to intrinsic) goals, which may "crowd out" (Frey, 1997) opportunities to meet their basic psychological needs. For example, an individual with high narcissistic tendencies might neglect his family in 
the pursuit of the accumulation of excessive wealth (Kasser \& Ryan, 1996), and in doing so may miss out on opportunities to satisfy his basic psychological need for relatedness. Moreover, when narcissistic individuals do pursue intrinsic goals, such as social relationships, they often do so to raise their own social status and not necessarily for the intrinsic benefits (e.g., intimacy, connection) that might otherwise enhance their well-being (Campbell, 1999; Horton \& Sedikides, 2009). Thus, individuals with higher narcissistic tendencies may not reap the benefits to well-being that are normally associated with pursuing intrinsic goals.

It is also possible that in the pursuit of extrinsic goals, individuals with higher narcissistic tendencies exhibit behaviours that compromise goal progress and well-being. For example, overt expressions of grandiosity or entitlement may evoke negative reactions in others, making it difficult for narcissistic individuals to achieve their goal of elite social status. This idea highlights an important limitation in the present research: goal attainment was not examined. Future research should include measures of goal attainment and investigate the discrepancy between the benefits that individuals with higher narcissistic tendencies believe they will receive from attaining extrinsic goals and the benefits that they actually do receive over time (e.g., Sheldon et al., 2010). The satisfaction or pride typically associated with attaining a goal (Bandura \& Locke, 2003) may be short-lived in the case of extrinsic goals (Niemiec et al., 2009), particularly among narcissists who require incessant external validation to maintain their sense of superiority (Gregg \& Sedikides, 2010). Importantly, this research should include objective measures of goal progress (e.g., GPA for academic goals, bodyweight for weight loss goals) since people with greater narcissistic tendencies tend to overinflate their sense of self-worth (Pincus, Cain, \& Wright, 2014) and, in a similar fashion, may provide inflated ratings of their goal attainment in a survey (Guedes, 2017).

\section{Methodological Considerations}

In addition to extending the findings of Abeyta and colleagues (2017), we also sought to address some of their methodological limitations. Most notably, we included assessed all of the intrinsic and extrinsic goal

6 This is not to say that researchers should never focus on any of the goals individually. In fact, this has been done quite frequently in the context of materialism (or the goal for financial success). This point here is directed at researchers components measured by the Aspirations Index (Kasser \& Ryan, 1996). As expected, we found that image (the omitted extrinsic goal) was positively associated with narcissistic tendencies and was highly correlated with the other extrinsic goals, indicating that its inclusion is indeed important when assessing the extrinsic goals construct. From a measurement perspective, it is important not to drop items (e.g., image, personal growth) arbitrarily6 (especially when all goal types are indeed assessed, as is the case with the original study), as this can inadvertently compromise the operationalization of the construct, bias the interpretation of results, and limits the generalizability of the findings or the ability to compare the findings to previous research (see Furr, 2011 for an in depth discussion of the issue with modifying validated measures, as well as Flake \& Fried, 2019 for a discussion on reproducible measurement practices).

Another measurement concern was how meaning in life was assessed. Abeyta and colleagues (2017) further modified the Aspirations Index by simply replacing the original instructions regarding perceived importance with how meaningful they perceived each goal to be. To us, these operationalizations of importance and meaning (at least how it was described in this particular measure) seemed quite redundant, which appears to be the case given their high correlation. Further concern is prompted by the observation that perceiving extrinsic (relative to intrinsic) goals as more meaningful was negatively related to eudaimonic well-being. Meaningfulness is a central component of eudaimonia and meaning in life is typically positively associated with eudaimonic well-being (Steger et al., 2008). Taking these two points into consideration, it is unclear whether Abeyta et al. (2017) actually assessed whether people with narcissistic tendencies derive any meaning from extrinsic pursuits in this particular study. Future research would benefit from more rigorous approaches to assessing meaning (e.g., using previously validated measures; Steger, Frazier, Oishi, Kaler, 2006).

An important limitation of both the present study and the original is the use of the NPI-16 as a measurement of narcissistic tendencies. Although we used the NPI-16 (Ames et al., 2006) as part of the direct replication of the original study, this measure is more reflective of grandiose narcissism, which typically is not strongly associated with psychological distress

who are interested in the broader construct of "intrinsic" and "extrinsic" goals, in which case all goal types should be assessed when possible. 
(Tritt, Ryder, Ring, \& Pincus, 2010). Thus, researchers should consider including measures of vulnerable narcissism (e.g., the hypersensitive narcissism scale; Hendin \& Cheek, 1997; the pathological narcissism inventory; Pincus et al., 2009; the short form of the five-factor narcissism inventory; Sherman et al., 2015). It is possible that vulnerable narcissism would exhibit a different pattern, as individuals with these traits score more highly on neuroticism and are more likely to report anxiety and depression than those with grandiose narcissism (Miller et al., 2011). Future research examining the relation between narcissism and goal pursuit can investigate these possibilities.

Finally, although the present study and its corresponding statistical code was pre-registered on the Open Science Framework prior to collecting any data, our research could have been further enhanced by using the registered report method (Chambers, 2013; Nosek \& Lakens, 2014). Registered reports highlight the importance of the research question and the quality of the proposed methodology by carrying out the peer review process prior to data collection. One of the notable benefits of this approach is that reviewers can suggest amendments to the proposed research methodology that can be incorporated before the study is conducted. This cooperation between the reviewers and the researchers helps to ensure that the most appropriate method is used to address the research question, thereby reducing the limitations of the study (e.g., our comment about the limitations of the NPI-16 and the suggestion of using a more comprehensive measure came from a reviewer; had we received that feedback in advance, it could have strengthened our research design rather than it now being a limitation of our work). We would thus strongly encourage researchers interested in conducting a replication attempt (like the one described in the current paper) to consider adopting the registered report format.

\section{Conclusion}

In the present study, we sought to replicate and extend Abeyta and colleagues' (2017) finding that people with greater narcissistic tendencies may derive positive benefits from pursuing extrinsic goals. While both the original study and our findings indicate that people with narcissistic tendencies may perceive materialistic pursuits as being more meaningful, here we actually included measures of well-being and found that valuing extrinsic goals was in turn negatively associated with subjective and eudaimonic well-being and positively associated with ill-being. Overall, these findings are consistent with self-determination theory, suggesting that the pursuit of extrinsic goals can be detrimental, even in cases where they may appear to be beneficial.

\section{References}

Abeyta, A. A., Routledge, C., \& Sedikides, C. (2017). Material meaning: Narcissists gain existential benefits from extrinsic goals. Social Psychological and Personality Science, 8, 219 228.

Ackerman, R. A., Donnellan, M. B., Roberts, B. W., \& Fraley, R. C. (2016). The effect of response format on the psychometric properties of the Narcissistic Personality Inventory: Consequences for item meaning and factor structure. Assessment, 23 203-220 https://doi.org/10.1177\%2F1073191114568113

Ames, D. R., Rose, P., \& Anderson, C. P. (2006). The NPI-16 as a short measure of narcissism. Journal of Research in Personality, 40, 440-450.

Bandura, A., \& Locke, E. A. (2003). Negative self-efficacy and goal effects revisited. Journal of Applied Psychology, 88, 87-99.

Campbell, W. K. (1999). Narcissim and romantic attraction. Journal of Personality and Social Psychology, 77, 1254-1270.

Chambers, C.D. (2013). Registered Reports: A new publishing initiative at Cortex. Cortex, 49, 609-610.

Deci, E. L., \& Ryan, R. M. (2000). The "what" and "why" of goal pursuits: Human needs and the self-determination of behavior. Psychological Inquiry, 11, 227-268.

Diener, E., Emmons, R. A., Larsen, R. J., \& Griffin, S. (1985). The satisfaction with life scale. Journal of Personality Assessment, 49, 71-75

Dittmar, H., Bond, R., Hurst, M., \& Kasser, T. (2014). The relationship between materialism and personal well-being: A meta-analysis. Journal of Personality and Social Psychology, 107, 879-924.

Eaton, W. W., Smith, C., Ybarra, M., Muntaner, C., \& Tien, A. (2004). Center for Epidemiologic Studies Depression Scale: Review and Revision (CESD and CESD-R). In M. E. Maruish (Ed.), The use of psychological testing for treatment planning and outcomes assessment: Instruments for adults (pp. 363 377). Mahwah, NJ: Lawrence Erlbaum Associates Publishers.

Frey, B. S. (1997). Not just for the money: an economic theory of personal motivation. Cheltenham, UK ; Brookfield, Vt: Edward Elgar Pub.

Furr, M. (2011). Scale construction and psychometrics for social and personality psychology. SAGE Publications Ltd.

Gregg, A. P., \& Sedikides, C. (2010). Narcissistic fragility: Rethinking its links to explicit and implicit self-esteem. Self and Identity, 9, 142-161.

Grouzet, F. M. E., Kasser, T., Ahuvia, A., Dols, J. M. F., Kim, Y., Lau, S., ... Sheldon, K. M. (2005). The structure of goal contents across 15 cultures. Journal of Personality and Social Psychology, 89, 800-816.

Guedes, M. J. C. (2017). Mirror, mirror on the wall, am I the greatest performer of all? Narcissism and self-reported and objective performance. Personality and Individual Differences, 108, 182-185.

Hayes, A. F. (2013). Introduction to mediation, moderation, and conditional process analysis: a regression-based approach. New York: The Guilford Press. 
Hendin, H. M., \& Cheek, J. M. (1997). Assessing hypersensitive narcissism: A reexamination of Murray's Narcism Scale. Journal of research in personality, 31, 588-599.

Horton, R. S., \& Sedikides, C. (2009). Narcissistic responding to ego threat: When the status of the evaluator matters. Journal of Personality, 77, 1493-1526.

Huta, V., \& Ryan, R. M. (2010). Pursuing pleasure or virtue: The differential and overlapping well-being benefits of hedonic and eudaimonic motives. Journal of Happiness Studies, 11, 735762.

Kasser, T. (2002). Sketches for a self-determination theory of values. In E. L. Deci \& R. M. Ryan (Eds.), Handbook of selfdetermination research (pp. 123-140). Rochester, New York: University of Rochester Press.

Kasser, T., \& Ahuvia, A. (2002). Materialistic values and wellbeing in business students. European Journal of Social Psychology, 32, 137-146.

Kasser, T., Cohn, S., Kanner, A. D., \& Ryan, R. M. (2007). Some costs of American corporate capitalism: A psychological exploration of value and goal conflicts. Psychological Inquiry, $18,1-22$.

Kasser, T., \& Ryan, R. M. (1993). A dark side of the American Dream: Correlates of financial success as a central life aspiration. Journal of Personality and Social Psychology, 65, 410-422.

Kasser, T., \& Ryan, R. M. (1996). Further examining the American Dream: Differential correlates of intrinsic and extrinsic goals. Personality and Social Psychology Bulletin, 22, 280-287.

Lee, S. Y., \& Seidle, R. (2012). Narcissists as consumers: The effects of perceived scarcity on processing of product information. Social Behavior and Personality: An International Journal, 40, 1485-1499.

Litman, L., Robinson, J., \& Abberbock, T. (2016). TurkPrime.com: A versatile crowdsourcing data acquisition platform for the behavioral sciences. Behavior Research Methods, 1-10.

MacKinnon, D. P., Krull, J. L., \& Lockwood, C. M. (2000). Equivalence of the mediation, confounding and suppression effect. Prevention Science, 1, 173-181.

Martin, B. A., Jin, H. S., O'Connor, P. J., \& Hughes, C. (2019). The relationship between narcissism and consumption behaviors: A comparison of measures. Personality and Individual Differences, 141, 196-199.

McGregor, I., \& Little, B. R. (1998). Personal projects, happiness, and meaning: On doing well and being yourself. Journal of Personality and Social Psychology, 74, 494-512.

Miller, J. D., Hoffman, B. J., Gaughan, E. T., Gentile, B., Maples, J., \& Keith Campbell, W. (2011). Grandiose and vulnerable narcissism: A nomological network analysis. Journal of personality, 79, 1013-1042.

Morf, C. C., \& Rhodewalt, F. (2001). Unraveling the paradoxes of narcissism: A dynamic self-regulatory processing model. Psychological Inquiry, 12, 177-196.

Niemiec, C. P., Ryan, R. M., \& Deci, E. L. (2009). The path taken: Consequences of attaining intrinsic and extrinsic aspirations in post-college life. Journal of Research in Personality, 43, 291306.

Nosek, B. A., \& Lakens, D. (2014). Registered reports: A method to increase the credibility of published results. Social Psychology, 45, 137-141.

O'Reilly, C. A., Doerr, B., \& Chatman, J. A. (2018). "See You in Court": How CEO narcissism increases firms' vulnerability to lawsuits. The Leadership Quarterly, 29, 365-378.
Pincus, A. L., Ansell, E. B., Pimentel, C. A., Cain, N. M., Wright, A. G., \& Levy, K. N. (2009). Initial construction and validation of the Pathological Narcissism Inventory. Psychological assessment, 21, 365-379.

Pincus, A. L., Cain, N. M., \& Wright, A. G. (2014). Narcissistic grandiosity and narcissistic vulnerability in psychotherapy. Personality Disorders: Theory, Research, and Treatment, 5, 439-443.

Rose, P. (2007). Mediators of the association between narcissism and compulsive buying: The roles of materialism and impulse control. Psychology of Addictive Behaviors, 21, 576-581.

Ryan, R. M., \& Deci, E. L. (2017). Self-determination theory: Basic psychological needs in motivation, development, and wellness. Guilford Publications.

Ryan, R. M., \& Huta, V. (2009). Wellness as healthy functioning or wellness as happiness: the importance of eudaimonic thinking (response to the Kashdan et al. and Waterman discussion). The Journal of Positive Psychology, 4(3), 202-204.

Ryan, R. M., Huta, V., \& Deci, E. L. (2008). Living well: A selfdetermination theory perspective on eudaimonia. Journal of happiness studies, 9, 139-170.

Sagiv, L., \& Schwartz, S. H. (2000). Value priorities and subjective well-being: direct relations and congruity effects. European Journal of Social Psychology, 30, 177-198.

Sheldon, K. M., Gunz, A., Nichols, C. P., \& Ferguson, Y. (2010). Extrinsic value orientation and affective forecasting: Overestimating the rewards, underestimating the costs. Journal of Personality, 78, 149-178.

Sheldon, K. M., \& Kasser, T. (1998). Pursuing personal goals: Skills enable progress but not all progress is beneficial. Personality and Social Psychology Bulletin, 24, 1319-1331.

Sheldon, K. M., \& Kasser, T. (2001). Goals, congruence, and positive well-being: New empirical support for humanistic theories. Journal of Humanistic Psychology, 41, 30-50.

Sheldon, K. M., \& Krieger, L. S. (2004). Does legal education have undermining effects on law students? Evaluating changes in motivation, values, and well-being. Behavioral Sciences \& the Law, 22, 261-286.

Sherman, E. D., Miller, J. D., Few, L. R., Campbell, W. K., Widiger, T. A., Crego, C., \& Lynam, D. R. (2015). Development of a Short Form of the Five-Factor Narcissism Inventory: The FFNI-SF. Psychological Assessment, 27, 11101116.

Steger, M. F., Kashdan, T. B., \& Oishi, S. (2008). Being good by doing good: Daily eudaimonic activity and well-being. Journal of Research in Personality, 42, 22-42.

Steger, M. F., Frazier, P., Oishi, S., \& Kaler, M. (2006). The Meaning in Life Questionnaire: Assessing the presence of and search for meaning in life. Journal of Counseling Psychology, $53,80-93$.

Sheldon, K. M., \& McGregor, H. A. (2000). Extrinsic value orientation and "the tragedy of the commons". Journal of personality, 68(2), 383-411.

Stucke, T. S., \& Sporer, S. L. (2002). When a grandiose self-image is threatened: Narcissism and self-concept clarity as predictors of negative emotions and aggression following ego-threat. Journal of Personality, 70, 509-532.

Tritt, S. M., Ryder, A. G., Ring, A. J., \& Pincus, A. L. (2010). Pathological narcissism and the depressive temperament. Journal of Affective Disorders, 122(3), 280-284.

Vansteenkiste, M., Duriez, B., Simons, J., \& Soenens, B. (2006). Materialistic values and well-being among business students: Further evidence of their detrimental effect. Journal of Applied Social Psychology, 36, 2892-2908. 
Vazire, S., Naumann, L. P., Rentfrow, P. J., \& Gosling, S. D. (2008). Portrait of a narcissist: Manifestations of narcissism in physical appearance. Journal of Research in Personality, 42, 1439-1447.

Waterman, A. S., Schwartz, S. J., Zamboanga, B. L., Ravert, R. D., Williams, M. K., Bede Agocha, V., ... Brent Donnellan, M. (2010). The questionnaire for eudaimonic well-being: Psychometric properties, demographic comparisons, and evidence of validity. The Journal of Positive Psychology, 5, 4161.

Watson, C., Clark, L. A., \& Tellegen, A. (1988). Development and validation of brief measures of positive and negative affect: The PANAS scales. Journal of Personality and Social Psychology, 54, 1063-1070.
Werner, K. M., \& Milyavskaya, M. (2018). We may not know what we want, but do we know what we need? Examining the ability to forecast need satisfaction in goal pursuit. Social Psychological and Personality Science.

Williams, G. C., Hedberg, V. A., Cox, E. M., \& Deci, E. L. (2000). Extrinsic life goals and health-risk behaviors in adolescents. Journal of Applied Social Psychology, 30, 1756-1771.

This pre-print was designed using the following template: Wiernik, B. M. (2019, October 11). Preprint templates. https://doi.org/10.17605/OSF.IO/HSV6A 